\title{
Object Recognition: Performance evaluation using SIFT and SURF
}

\author{
Ritu Rani \\ M.Tech Scholar \\ Deenbandhu Chhotu Ram \\ University of Science and \\ Technology,Murthal
}

\author{
Surender Kumar Grewal \\ Associate Professor \\ Deenbandhu Chhotu Ram \\ University of Science and \\ Technology,Murthal
}

\author{
Kuldeep Panwar \\ Assistant Professor \\ HMR Institute of Technology \\ and Management, Hamidpur \\ New Delhi
}

\begin{abstract}
Object Recognition has become one of the most attractive areas of research for most of the scientists over the past few decades. Object recognition has extensive applications in numerous areas of interest. In this paper, the importance of object recognition in different applications has been highlighted. The very famous and impressive technique by David Lowe which is Scale Invariant Feature Transform (SIFT) has been implemented for object recognition and an attempt has been done to compare the results obtained from it with the another very important technique called Speeded-Up Robust Feature Transform (SURF) to conclude with certain interesting results.
\end{abstract}

\section{KEYWORDS:}

Object recognition, features, applications, SIFT, SURF

\section{INTRODUCTION}

Object Recognition is one of the core areas of research in computer vision. A lot of work and interest is been shown in this field since it has proved to be very useful for a number of applications whether face recognition [1], iris or fingerprint recognition, augmented reality and Robotic manipulations, military, medical diagnosis, vehicle counting, surveillance and last but not the least for security purposes[2].

Object recognition is basically concerned with the recognition of 3 dimensional objects from image data. It also involves the approximation of the positions and orientations of the recognized objects in the $3 \mathrm{D}$ world. Research process is in this field is going on since few decades and noteworthy progress has been done in this direction during all this time [3].

Basic Object recognition systems [4] involve extraction of features and then matching of these features with the features calculated and stored in the database. Features are basically the 'keypoints' which can uniquely define the whole object i.e. the features should be able to give the most of the information about the object/data. Features can be patches, edges, corners. When all images are similar in nature (same scale, orientation, etc) simple corner detectors [5] can work. But when there are images of different scales and rotations, then there is need to use some very advanced techniques which can help recognize the objects under all these constraints of different scaling, orientations, illuminations and occlusion.
Thus, in this paper an introduction to two very effective techniques has been given

- $\quad$ Scale Invariant Feature Transform

- $\quad$ Speeded-Up Robust Feature Transform

Here is a brief about the organization of this paper. The section 2 will talk about the David Lowe's Scale Invariant Feature Transform Technique and the section 3 will talk about the Herbert Bay's Speeded-Up Robust Feature Transform. In section 4 a brief review about the implementation and the data analysis of these techniques on certain images taken using Samsung Galaxy note-2, 8 MPixel camera is given. In section 5 a glimpse of certain interesting results and conclusion has been given.

\section{SCALE INVARIANT FEATURE TRANSFORM}

David Lowe in 1991 [6] gave this Scale Invariant Feature Transform (SIFT) wherein he implemented this technique for object recognition. SIFT has now been successfully implemented in number of other applications [7] as well such as fingerprint recognition [8], face recognition [9] [10], ear recognition [11], real-time hand gesture recognition [12], iris recognition [13]. SIFT provides us with features which are robust to illumination changes, scaling, orientation, occlusion etc.

SIFT [14] is quite an involved algorithm and thus it can be broken down into steps as follows:

- Constructing a scale space

- LoG Approximation

- Finding keypoints

- Get rid of bad key points

- Assigning an orientation to the keypoints

- Generate SIFT features

Thus, SIFT is a method for extracting distinctive invariant features from images that can be used to perform reliable matching between different images of the same object or scene. 


\section{SPEEDED-UP TRANSFORM}

ROBUST

SURF [15] is also popularly known as approximate SIFT. It uses integral images and efficient scale space construction for the efficient generation of keypoints and descriptors. SURF basically involves two stages

- Keypoint detection

- Keypoint description

In the first stage, instead of using Difference of Gaussian like in SIFT, integral images are used which allow the fast computation of approximate Laplacian of Gaussian(LoG) images using a box filter. The computational cost of applying the box filter is independent of the size of the filter because of the integral image representation. Determinants of the Hessian matrix are then used to detect the keypoints. In order to be

Given below is the input image taken from the database. invariant to rotation, it calculates the Haar-wavelet responses in $\mathrm{x}$ and $\mathrm{y}$ direction.

\section{IMPLEMENTATION AND DATA ANALYSIS}

The algorithm has been implemented for the MATLAB environment. A small database of certain images has been created using SAMSUNG GALAXY NOTE-2 Camera with 8.0 MP resolution. All the images correspond to daylight scenes. The original images were resized to a lower resolution of approximately $457 \times 630$ pixels so that the algorithms chosen can process them more efficiently. The experiments are performed on Intel Core i-3 3210, $2.3 \mathrm{GHz}$ processor and 4 GB RAM with windows 7 as an operating system. Features are detected in both images using SIFT and SURF algorithm.

\subsection{FEATURE DETECTION USING SIFT}

The features of the image are extracted using Scale Invariant Feature Transform

Figure 1. The features of the database image are extracted using SIFT

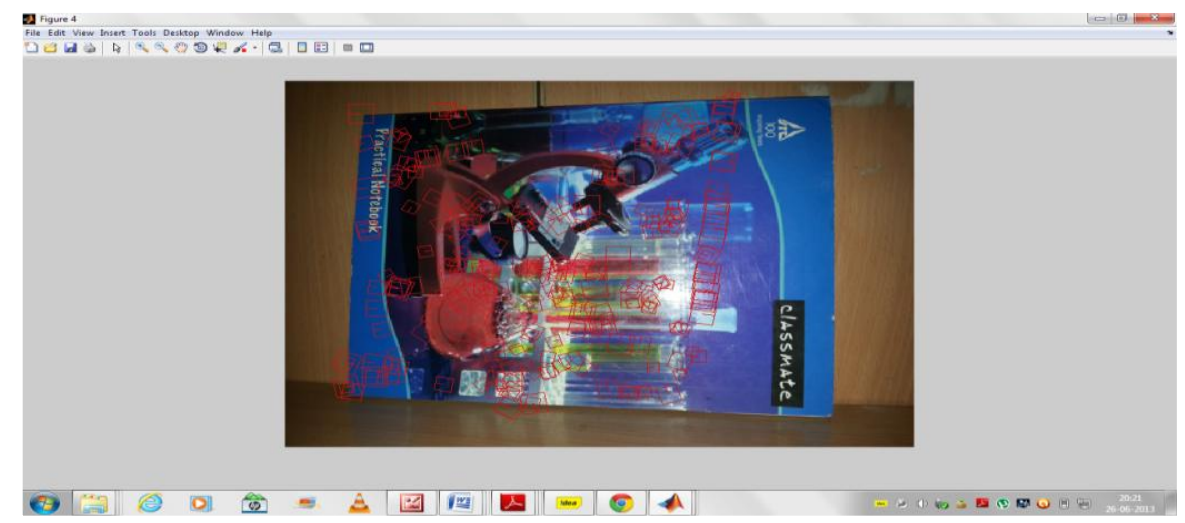

Figure 2. The input image is given and the features are extracted using SIFT

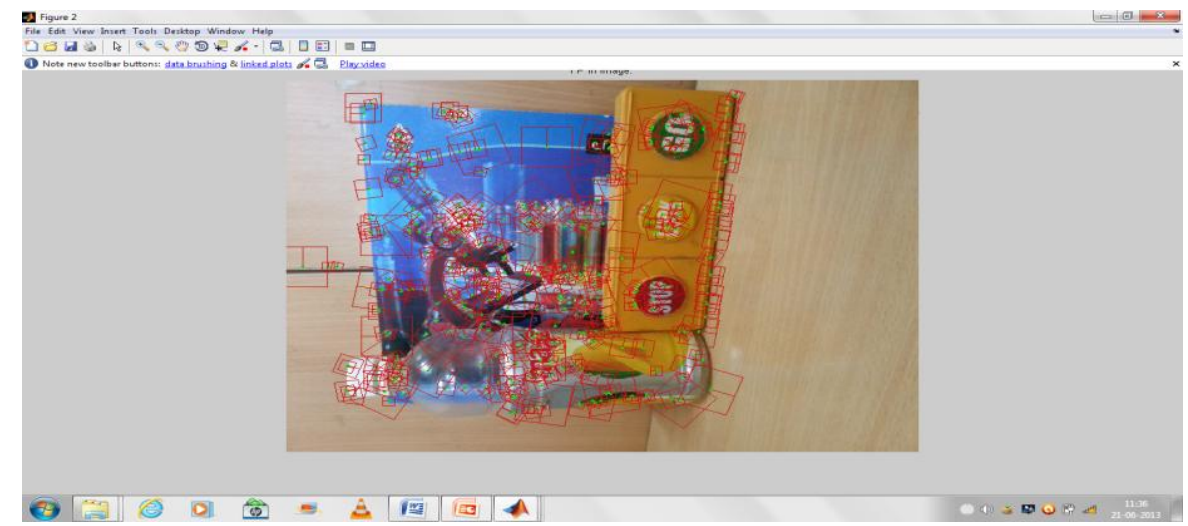




\subsection{FEATURE DETECTION USING SURF}

Given below are the input images taken from the database.
Features are extracted from the input image using the Speeded- Up Feature Transform.

Figure 3. The features of the database image are extracted using SURF

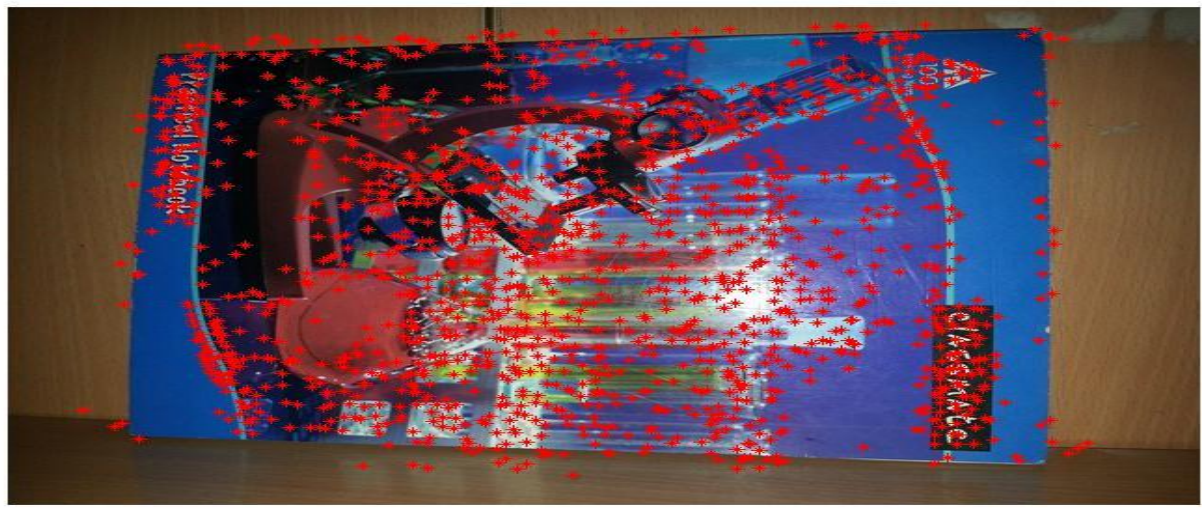

Figure 4. The input image is given and the features are extracted using SURF

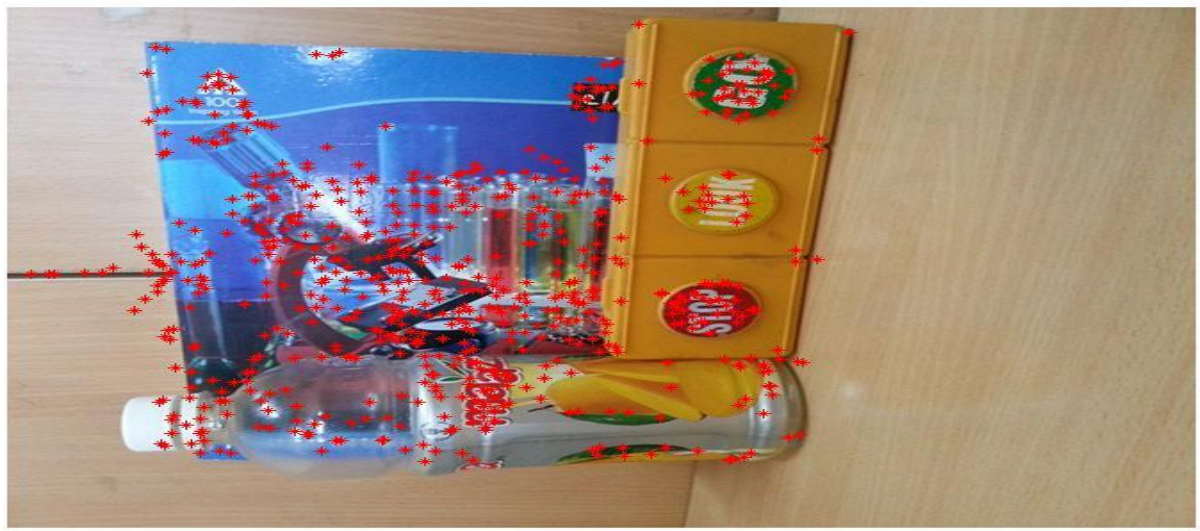

\subsection{FEATURE MATCHING USING SIFT}

Figure 5. The register in the input image is been matched with the register in the database image and it is been recognized using SIFT

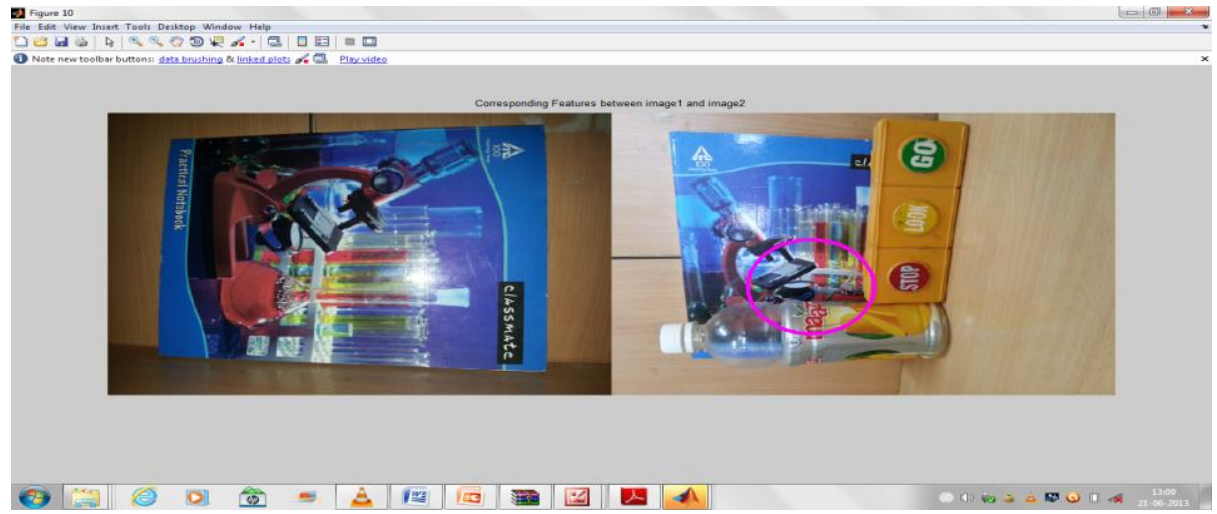




\subsection{FEATURE MATCHING USING SURF}

Figure 6. The register in the input image is been matched with the register in the database image and it is been recognized using SURF.

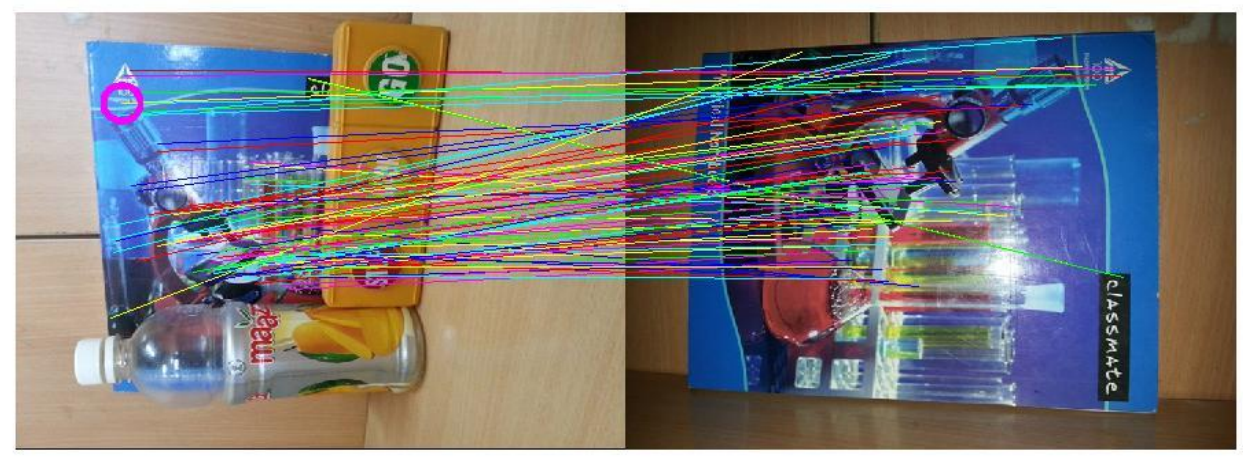

\section{RESULTS AND CONCLUSION}

SIFT and SURF has been implemented on the images and the results below can show that these techniques SIFT and SURF both can recognize objects under various constraints.

\subsection{RESULTS OBTAINED WITH SIFT}

Figure 7. The bottle is detected in the input image even after different orientation using SIFT

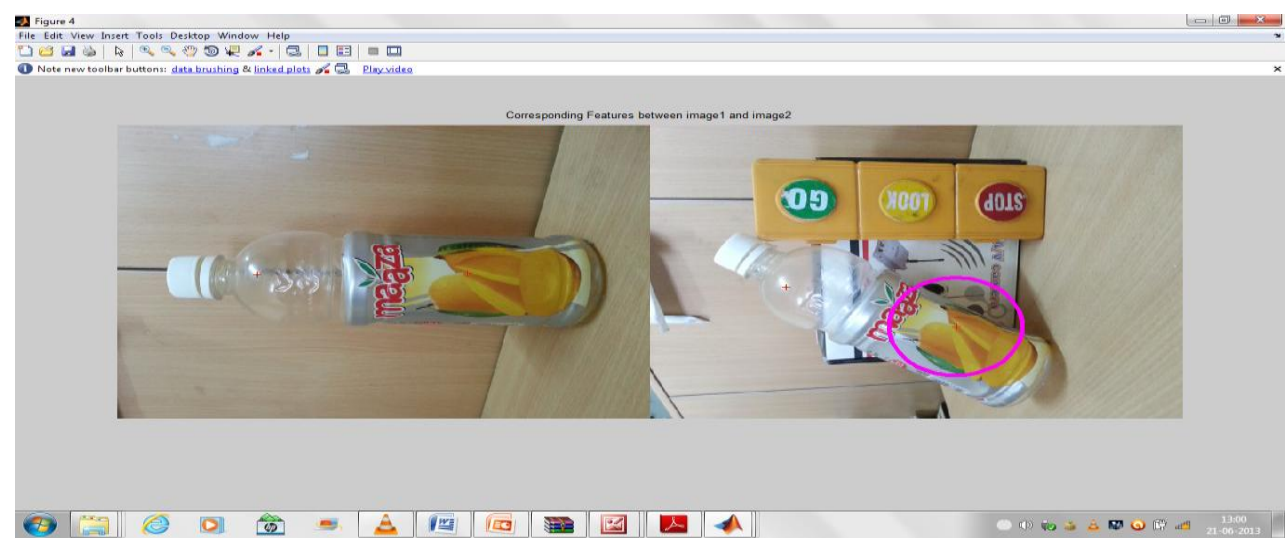


Figure 8. The yellow box is detected in the input image with different scaling and orientation and illumination too using SIFT

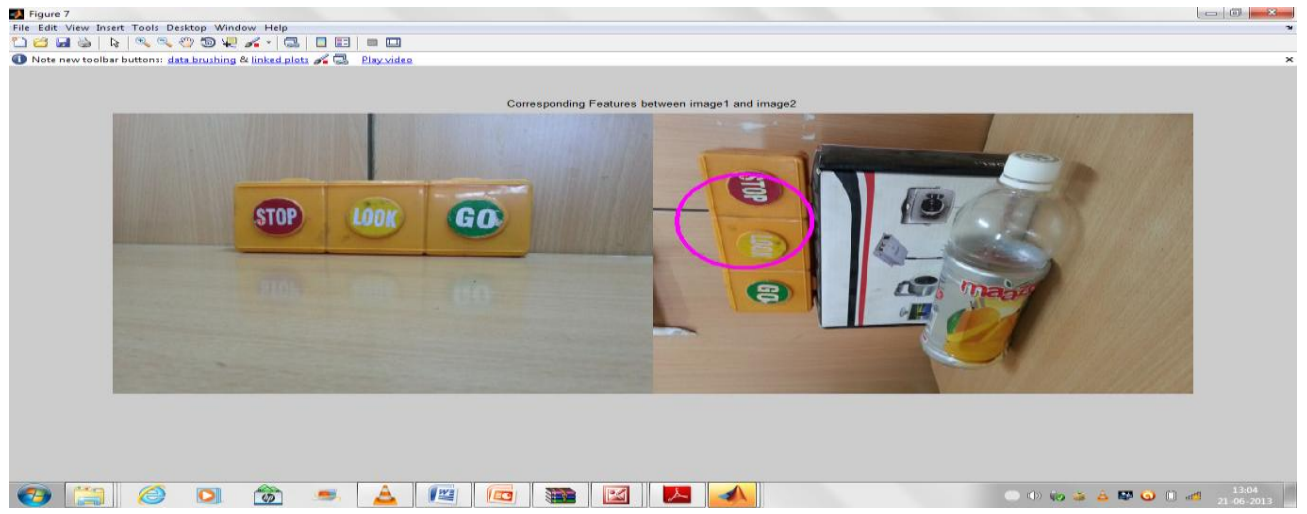

Figure 9.The box is detected in the input image even after different illumination, orientation using SIFT (IMAGE 1)

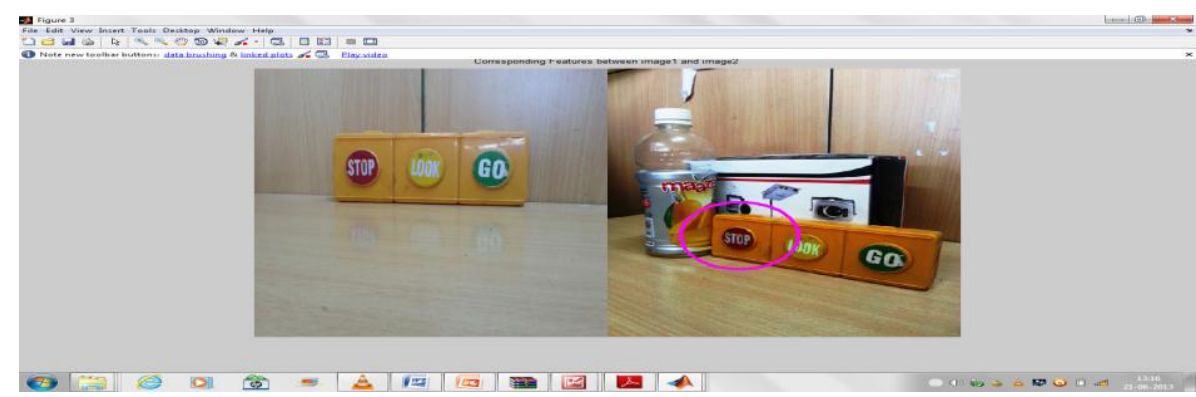

Figure 10. The box is detected in the input image even after different scaling using SIFT

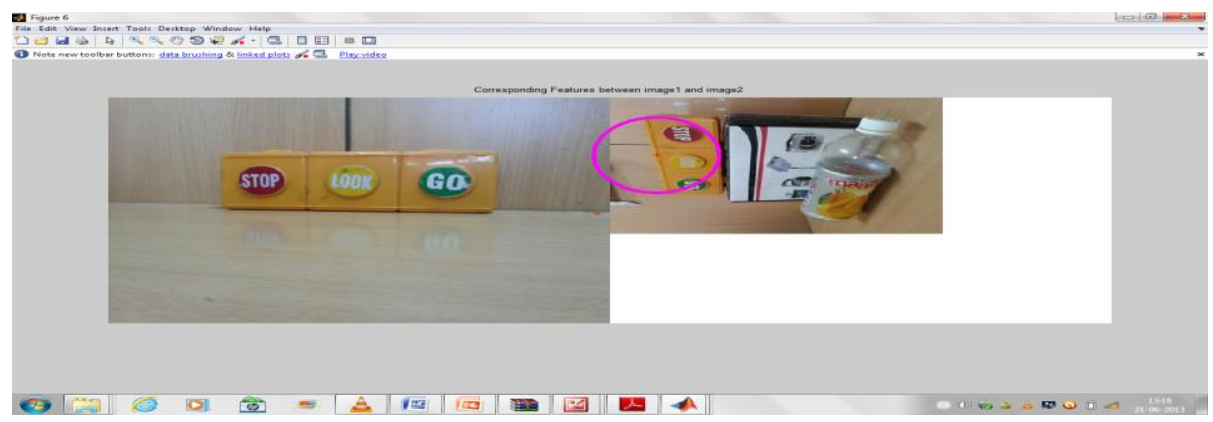


Figure 11. The register is been detected even after occlusion using SIFT (IMAGE II)

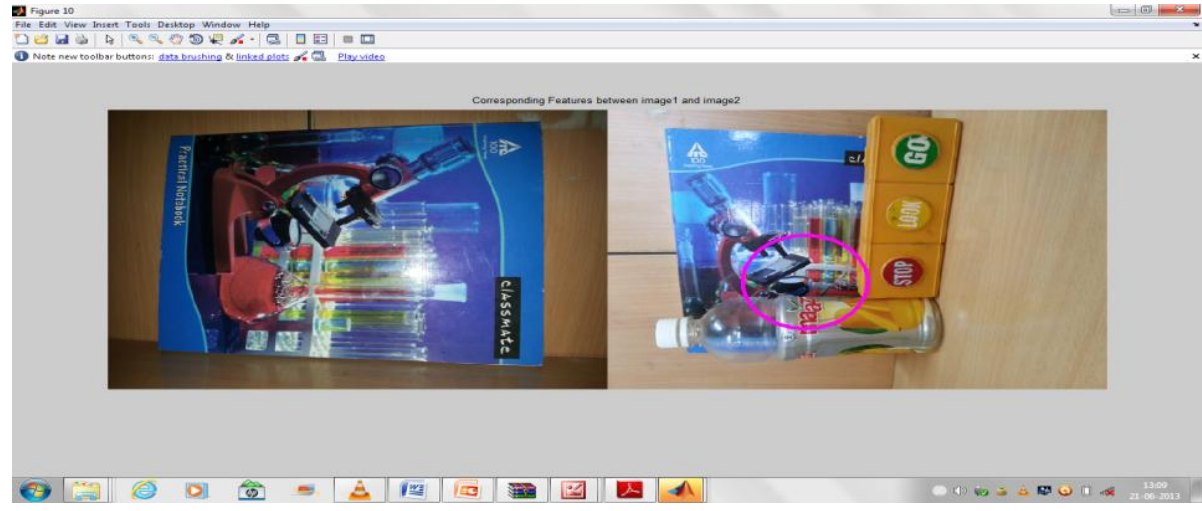

\section{RESULTS OBTAINED WITH SURF}

Figure 12. The box is detected in the input image even after different illumination, orientation and somewhat different scaling using SURF (IMAGE I)

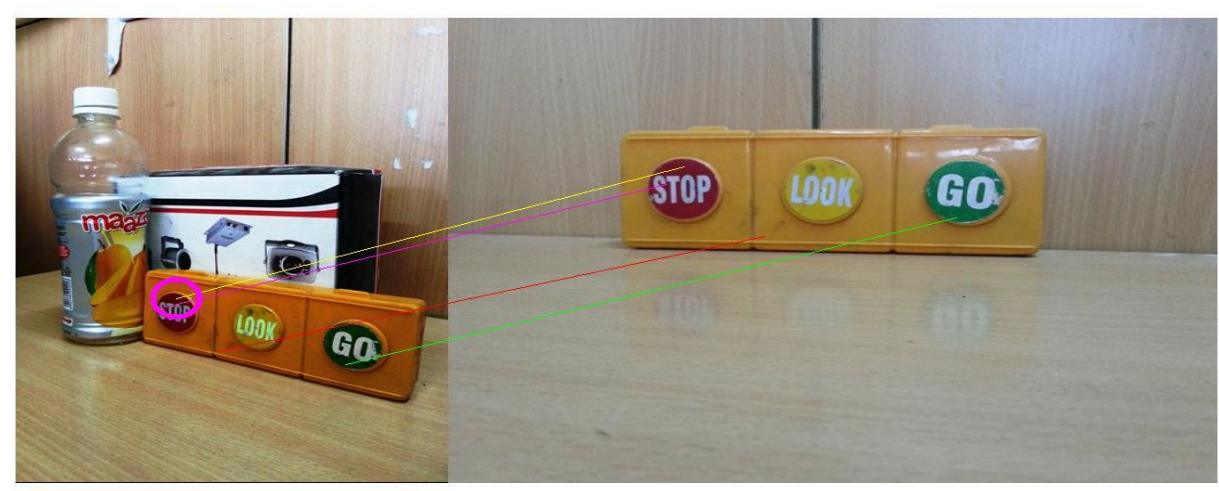


Figure 13. The white box is been matched and detected even under occlusion and different orientation using SURF

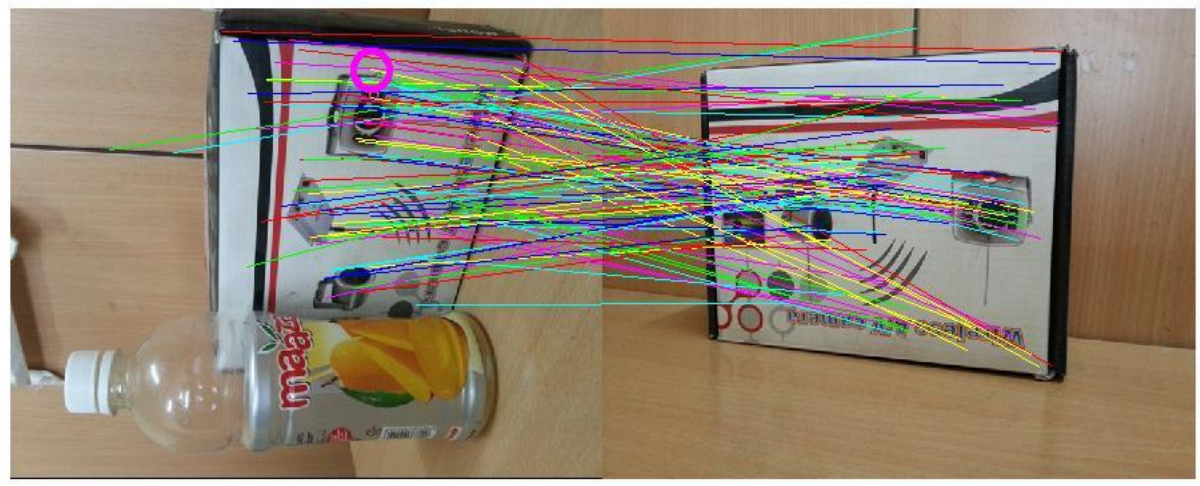

Figure 14. The register is been matched and detected even under occlusion and different illumination using SURF (IMAGE II)

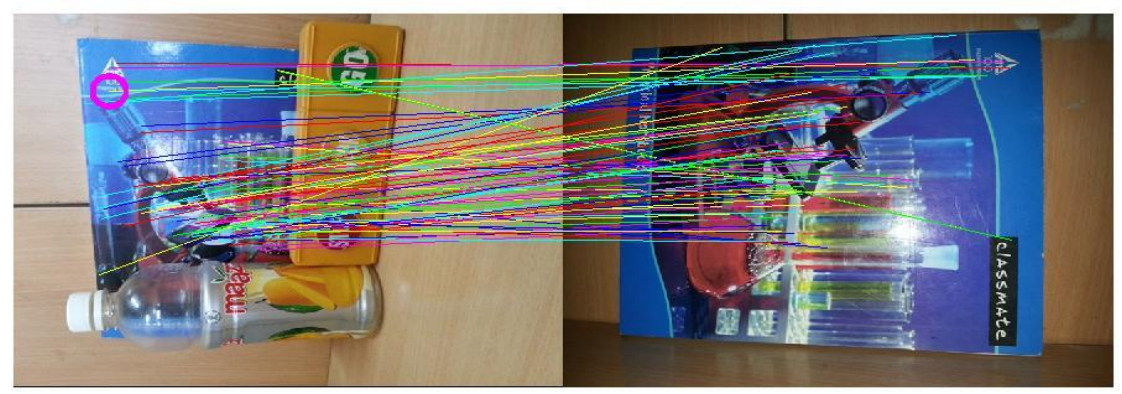

\section{COMPARISON BETWEEN SIFT} AND SURF

SIFT and SURF has been implemented on the given images in the database. These two techniques are compared mainly on two parameters

Figure 15 : Given below are the few images from database named 1,2,3,4 respectively

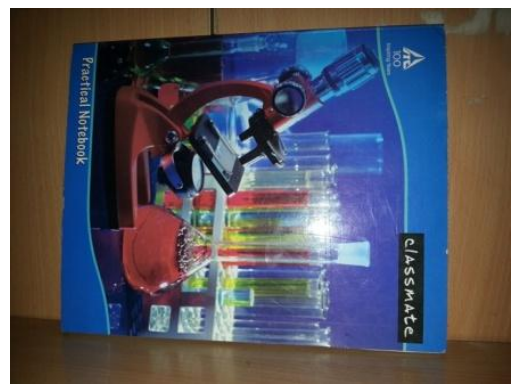

Image 1

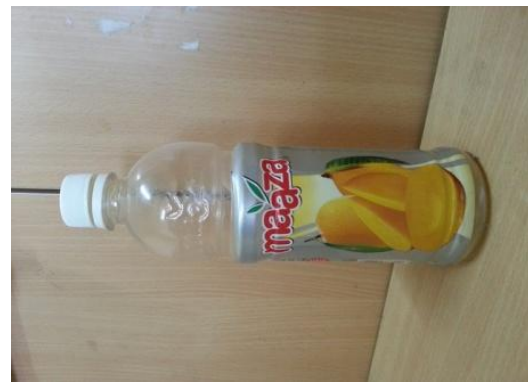

Image 2 


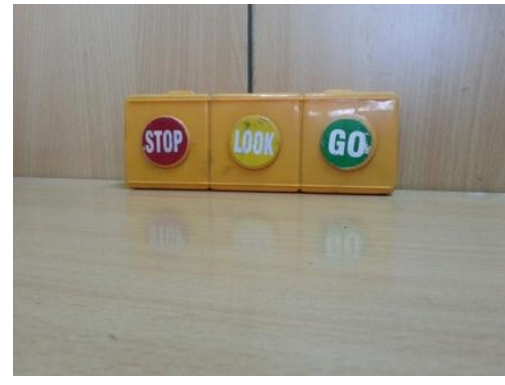

Image 3

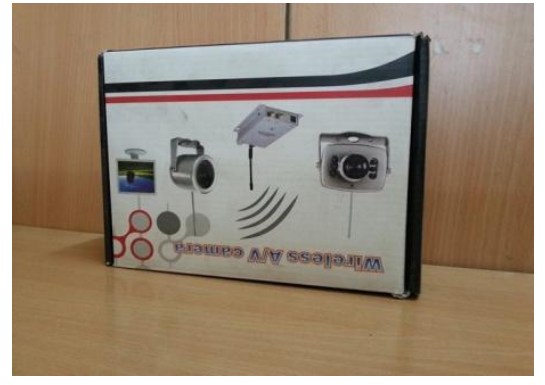

Image 4

Table 1 Table showing the number of features using SIFT and SURF

\begin{tabular}{|l|l|l|l|}
\hline \multicolumn{4}{|c|}{ NUMBER OF FEATURES } \\
\hline S.No. & IMAGE & SIFT FEATURES & SURF FEATURES \\
\hline 1 & 1 & 890 & 750 \\
\hline 2 & 2 & 870 & 770 \\
\hline 3 & 3 & 900 & 740 \\
\hline 4 & 4 & 865 & 755 \\
\hline
\end{tabular}

The given table gives the number of features detected using SIFT and SURF implemented on four images 1,2,3,4 taken from the database. From the table it can seen that the number of features extracted from SIFT is more than the features extracted from the SURF. The table below gives the execution time or the run time required for execution using SIFT and SURF. From the table it can seen that the execution time or the run time required in the SURF is less than the SIFT.

Table 2. Table showing the time taken for execution in SIFT and SURF

\begin{tabular}{|l|l|c|c|}
\hline \multicolumn{4}{|c|}{ TIME TAKEN FOR EXECUTION (SEC) } \\
\hline S.No. & IMAGE & SIFT & SURF \\
\hline 1 & IMAGE I & 45.291518 & 6.622343 \\
\hline 2 & IMAGE II & 96.850186 & 12.055012 \\
\hline
\end{tabular}

The images in figure 9 and figure 12 are named as Image I. The images in figure 11 and figure 14 are named as Image II. The SIFT and SURF has been applied on Image I and Image
II and the time taken for execution has been noted for both these.

Let's plot two graphs also to show this comparison between these two algorithms.

Figure 16. Graph comparing the number of features detected using SIFT and SURF

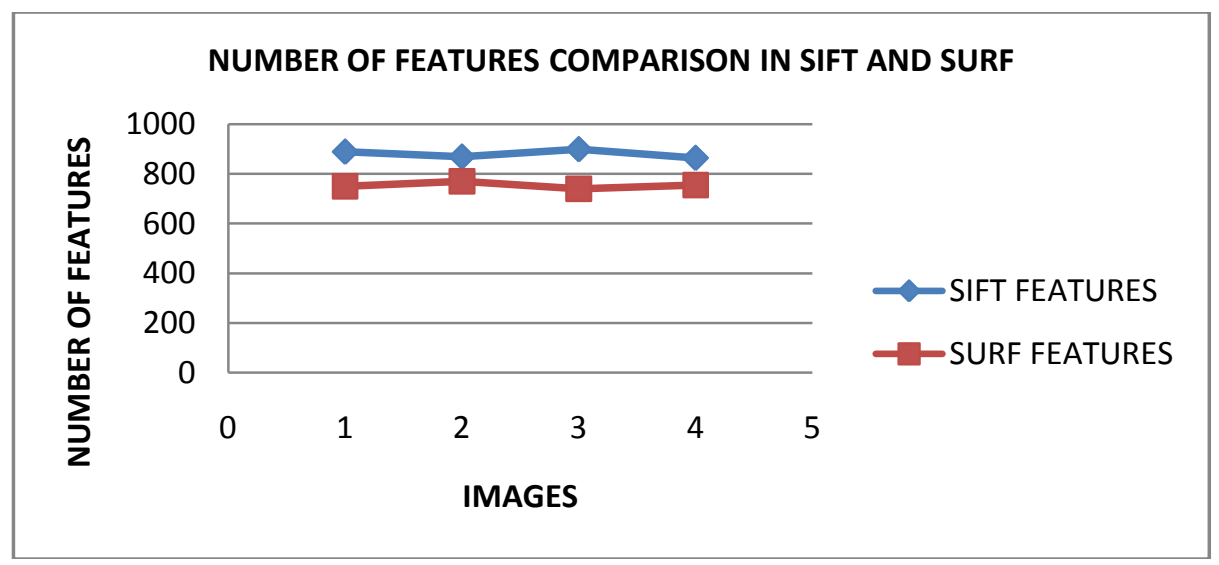


Figure 17. Graph comparing the time constraint in both SIFT and SURF

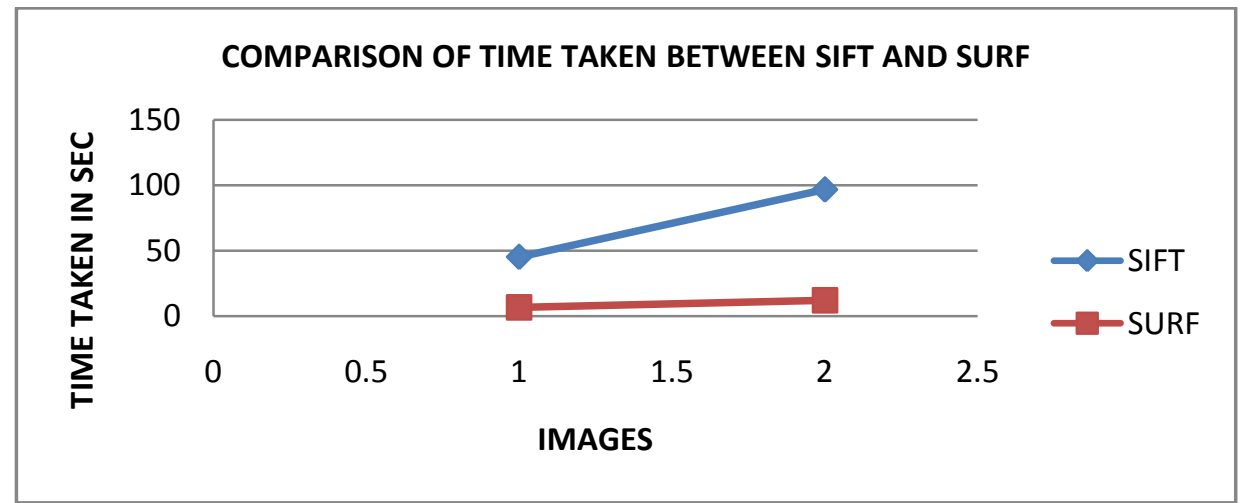

\section{CONCLUSION}

Thus, from both the graphs and the table we can conclude that the numbers of features detected using SIFT is more than that of SURF. But the execution time for SURF is less than the SIFT.

\section{REFERENCES}

[1] Rajeshwar Dass, Ritu Rani, Dharmender Kumar, "Face Recognition Techniques: A Review", International Journal of Engineering Research and Development" Volume 4, Issue 7 (November 2012), PP. 70-78

[2] Ritu Rani, Surender Kumar Grewal, "A Comprehensive Survey of Object Recognition Techniques", in National Conference on Contemporary Techniques and Technologies in Electronics Engineering at D.C.R.U.S.T Murthal, on March 14 $4^{\text {th }}, 2013$.

[3] J.Ponce, M. Hebert, C. Schmid, and A. Zisserman, editors. "Toward Category-level Object Recognition", SpringerVerlag, Volume 4, 2007.

[4] S. Dickinson,in: E. Lepore and Z. Pylyshyn , "What is Cognitive Science?", Basil Blackwell publishers, 1999, PP 172-207.

[5] Harris, C. and Stephens, " A combined corner. and edge detector", In Fourth Alvey Vision Conference, Manchester, UK, PP. 148-151,1988.

[6] Lowe, David G. (1999). "Object recognition from local scale-invariant features". Proceedings of the International Conference on Computer Vision. PP. 1150-1157, 1999.
[7] Ritu Rani, Surender Kumar Grewal, Indiwar, "Implementation of SIFT in various applications", International Journal of Engineering Research and Development”, Volume 7, Issue 4 ,PP. 59-64, 2013.

[8] Unsang Park, Sharath Pankanti, A. K. Jain, "Fingerprint Verification Using SIFT Features", SPIE Defense and Security Symposium, Orlando, Florida, 2008.

[9] Mohamed Aly, "Face Recognition using SIFT Features", Technical report, Caltech, California Institute of Technology USA, 2006.

[10] Geng C., Jiang X., "SIFT Features for Face Recognition", IEEE Conference CSIT, PP 598-602, 2009.

[11] Hunny Mehrotra, Phalguni Gupta, and Jamuna Kanta Singh, Dakshina Ranjan Kisku, “ SIFT-based Ear Recognition by Fusion of Detected Keypoints from Color Similarity Slice Regions” 2009.

[12] Nasser Dardas , "Real-time Hand Gesture Detection and Recognition for Human Computer Interaction” Technical Report, University of Ottawa, 2012.

[13] Fernando Alonso-Fernandez, Pedro Tome-Gonzalez, Virginia Ruiz-Albacete, Javier Ortega-Garcia, "Iris Recognition Based on SIFT Features",Biometric Recognition Group- AVTS, 2009.

[14] Lowe, "Object recognition from local scale-invariant features", The proceedings of the seventh IEEE International Conference on Computer Vision, PP 1150$1157,1999$.

[15] Herbert Bay, Andreas Ess, Tinne Tuytelaars, Luc Van Gool "SURF: Speeded Up Robust Features", Computer Vision and Image Understanding (CVIU), Vol. 110, No. 3, PP. 346--359, 2008. 